\title{
Comparison between Kit-Build and Scratch-Build Concept Mapping Methods in Supporting EFL Reading Comprehension
}

\author{
Mohammad Alkhateeb****, Yusuke Hayashi*, Taha Rajab*** and Tsukasa Hirashima*
}

(Received 19 June 2014 and accepted in revised form 13 February 2015)

\begin{abstract}
Reading comprehension is a rather difficult task for students in all the stages of study, especially when they are reading text in a foreign language. In the EFL (English as a foreign language) context, reading is one of the most intriguing and most common research topics. This study investigates the effects of teaching EFL reading comprehension to Japanese students through the Kit-Build concept mapping (KB-mapping) method. In doing so, we have conducted an experiment to compare the student comprehension with KB-mapping and Scratch-Build Concept Mapping (SC-mapping) methods. We gave participants a comprehension test (CT) and a delayed comprehension test (DCT; two weeks after using the mapping methods). By comparing the results of the CT and the DCT for the two conditions groups, we found that using KB-mapping was no better than using SC-mapping for participants understanding the text just after using the methods, but KB-mapping led to better effects in recalling and remembering of the text after a while.
\end{abstract}

Keywords: Technology-Enhanced learning, Kit-Build concept map system, reading comprehension, EFL

\section{Introduction}

In language learning contexts, reading comprehension is an important learning activity that requires a special ability from the learner to reap its benefits ${ }^{(1)}$. Reading comprehension poses many challenges since learners may experience such issues as slow reading, insufficient vocabulary comprehension and poor recalling ${ }^{(2)}$. Researchers have always tried to support this learning activity by proposing various methods or strategies ${ }^{(3-6)}$. The main goal is to boost comprehension skills in the target subject area. When these methods or strategies are deployed in a language course, the main aims are to improve student reading comprehension of the text and to contribute to the acquisition of the Target Language ${ }^{(7)}$.

Reading comprehension in an EFL context is a special case of reading comprehension; it is a complex, dynamic, multi-componential and multi-dimensional task in the learning process. It is a continuous process of multiple interactions between the readers' background knowledge in their Mother Language (ML) and the

\footnotetext{
* Graduate School of Engineering, Hiroshima University, Japan

**Faculty of Mechanical and Electrical Engineering, Tishreen University, Syria

***Department of Languages, HLSS, Manchester Metropolitan University, UK
}

knowledge exposed to in the Target Language (here English). Broadly speaking, the reading comprehension of EFL is the same as the ML reading comprehension but it is slower and less successful than ML reading ${ }^{(8)}$. This can be explained by the fact that the reading process is dependent on many factors such as the level of the readers' language proficiency, the subject matter of the text, text difficulty and task demands.

In this research, we are trying to support this complex learning task by using our Kit-Build concept mapping (KB-mapping) method ${ }^{(9)}$. We have been developing learning tools to help both students and teachers within the learning process. One of these tools is our KB-map. We have found it a very useful tool for learning the sciences for students in their $\mathrm{ML}^{(9)}$. Equally, we have found that this tool yielded good results for teachers and students. We note that the KB-map is a special kind of concept map, and using it requires a lot of concentration in order to recognize the two concepts that can be connected by a relation.

In this research, we are proposing the KB-mapping method as a supportive tool for the reading comprehension task by enhancing learners' performance in the reading task, as a future research topic the use of this method to develop the learners' reading ability will be a very attractive one. This research is preliminary and we have not finished it yet, so we cannot confirm the rela- 
tion between the enhancement of performance and the development of ability. We think that the question "Can learners get higher marks in the situation where the KB method support has faded?" should be one of research questions for our future studies.

Our previously conducted experiments in the $\mathrm{ML}^{(19-21)}$ showed that the KB-mapping method has good effects for performing tasks that require a high cognitive load, as an example learning science in elementary school $^{(20)}$, and that require memory retention ${ }^{(19)}$. In the latter study, we showed that the KB-mapping method has the same level of memory retention as the Scratch Concept mapping (SC-mapping) method. Also in previously conducted experiments in $\mathrm{EFL}^{(17,18)}$, we showed that the KB-mapping method is good as the underlining method just after use, but it has a better effect in recalling after some time has passed.

In the present research, we tried to investigate the effects of using KB-mapping in comprehending English text and recalling it after two weeks. We conducted an experiment to confirm the efficiency of the KB-mapping method in the learning tasks that require a high cognitive load such as EFL reading comprehension. We designed an experiment to compare SC-mapping for reading comprehension with our KB-mapping method. SCmapping is a flexible method that may be tailored to fit various types of information, and different skill levels. Also SC-mapping is a popular method to help learners in raising their reading comprehension ${ }^{(10)}$.

\subsection{EFL reading comprehension}

'Comprehension' as a concept is defined as "the ability to understand something" in the Oxford Dictionary, the definition in the Cambridge Dictionary is "the ability to understand completely and be familiar with a situation, facts, etc." For the purpose of this research, 'reading comprehension' will be defined as "a learner's ability to understand completely and memorize the important information that is included in the text that he/she is reading". The definition necessarily includes the level of understanding of a text/message. Such an understanding comes from the interaction between the written words and how they trigger knowledge outside the text/message ${ }^{(11)}$.

The effects of using a learning method on the learning ability of the learner, from the long-term viewpoint, are not easy to investigate, and a special environ- ment is needed to keep the fairness of experimental conditions, so in this research, we tried to investigate the effects in the learning performance for every session.

Reading comprehension is a difficult task for students at all stages of study, especially when they are reading texts in a foreign language. Therefore, EFL reading has attracted attention in the teaching and learning field. Many researches have proposed methods, techniques and strategies to help students improve their reading abilities. These attempts have yielded significant improvements in students' comprehension just after using the proposed methods or strategies ${ }^{(3-6)}$. To mention as examples, there are the Selective Underlining Strategy, Note-Taking Skills Reading(6), and SQ3R (Survey, Question, Read, Recite, Re-view) ${ }^{(12)}$. The newest strategy that has been proposed to support the reading comprehension task is Graphic Organizers (GOs) which is a reading strategy used both in the teaching and learning of languages ${ }^{(13)}$. Examples of GOs are Story Maps, matrix, Semantics Maps, Knowledge Maps and SC-maps ${ }^{(13)}$.

Several investigations of reading comprehension strategies have specifically addressed challenges related to reading expository text. Positive outcomes have been found for students who were taught strategies to help them identify the main idea ${ }^{(3-6,12,13)}$.

In general, reading comprehension has as its essential task to distinguish the important information in the text; to find the important information means not only to find the words, but more importantly to find the links and relations (structure). In other words, the distinguishing task is not only to pick out words (selection process) from the text, but also to recognize the relations (structuring process) between the words to complete the whole text structure.

Many studies ${ }^{(21-25)}$ have indicated that learners' performance in the structuring process has a stronger influence on the learners' comprehension than the performance in the selection process. Moreover, if learners fail to select the necessary information, it is impossible for them to recover from the failure. Based on these considerations, Armbruster ${ }^{(26)}$ suggested that reducing a learner's load in the selection process and letting the learner focus on the structuring process are promising ways to improve the learner's comprehension of a lecture).

\subsection{Scratch concept mapping method}

SC-mapping is one of the newest strategies used to 
support the reading comprehension learning task and it gives good effects on reading comprehension of EFL students $^{(10,14)}$. SC-maps are visual representations of knowledge which can be employed as a learning strategy by the learners to find the relationship between current knowledge and new information ${ }^{(14)}$. Research has confirmed that EFL students who used concept mapping gained high scores in reading comprehension ${ }^{(14)}$. Also there are many studies of students learning English reading through the use of concept maps showing that the concept mapping or semantic mapping technique could improve the students' reading comprehension because they could understand the text more easily through the concept map ${ }^{(10,14,15)}$.

SC-mapping provides students with opportunities to become actively involved in their learning while linking knowledge to long-term memory. Through the use of concept maps, students have opportunities to organize their thoughts in a concrete and/or graphic/visual format, while connecting concepts and linking prior knowledge to new knowledge ${ }^{(10,14-16)}$. Related concepts become connected rather than fragmented. Concept maps also provide students with opportunities to think about their own thinking as they reflect on their conceptual understandings. The process of map drawing has a positive impact on students' awareness of the reading process and they can have more control over reading comprehension in English by visually representing what is conveyed in the texts they read ${ }^{(14,16)}$.

In general, using the SC-mapping method has many advantages in the reading comprehension process, it helps students brainstorm and generate new ideas. Moreover, it encourages students to discover new concepts and the propositions that connect them, it allows students to more clearly communicate ideas, thoughts and information, and it enables students to gain enhanced knowledge of any topic and evaluate the information. All of these advantages improve the students' comprehension of text and allow them to recall the comprehended information after a while.

\subsection{Purpose of this research}

We have been developing learning tools to help both students and teachers in the learning process. One of these tools is the KB-map, which is a special kind of SC-map and, we have conformed its high efficiency in many fields of learning such as reading comprehension of EFL students ${ }^{(17)}$; moreover, it has effects in the remembering and recalling of the comprehended text after some time has passed ${ }^{(18-21)}$. Also we found it was very useful for learning sciences by students in their $\mathrm{ML}^{(9)}$. Moreover, some of our studies suggested that KBmapping is a promising tool to estimate learners' understanding in classrooms by using diagnosis and feedback with the KB-map ${ }^{(19,20)}$. It has good effects for the teachers and students.

In our previous research we found that the KBmapping method has almost the same efficiency as SCmapping method for a simple reading task in ML, also we found that it provides good support for performing tasks that require a high cognitive load. As we explained before, EFL reading comprehension exerts a bigger cognitive load to learners than ML reading comprehension does. So in this research we assumed that the KBmapping method has some advantages in supporting learners' performance in EFL reading comprehension in comparison with SC-mapping method.

The task to build a SC-map can be divided into two sub-tasks: 1) the "segmentation task" in which it is necessary to pick out the important words in the text and find the relations between them (i.e., to find every part of the important information); and 2) the "structuring task" in which the extracted parts are integrated into a $\mathrm{map}^{(27)}$. From this viewpoint, in the closed-end approach, for the task of building the KB-map: the picking out part is replaced by providing a kit (all the important concepts and relations), and the finding the relations part is replaced by the recognition task of the parts; finally, the structuring task is the same for both mapping methods. Therefore, learners are relieved of the burden of the selection and they are able to concentrate on the structuring task. Moreover, if learners fail to pick out necessary concepts and relations, it is impossible for them to recover from the failure. Based on these considerations, we felt that reducing the load of learners in the selection process and letting learners focus on the structuring process are promising ways to improve their comprehension.

SC-mapping is a very effective method to support the EFL students' reading comprehension task, and it is effective for comprehending and recalling the text. As we mentioned before the KB-map is a special kind of SCmap, and in this research, we are trying to use the KBmapping method to support the reading comprehension of EFL students, and to compare it with the SC-mapping 
method to confirm the efficiency of KB-mapping in the comprehension and recalling of English text.

\section{Kit-Build Concept Mapping Method}

While the SC-mapping and KB-mapping allow learners to organize ideas and determine the relations between them, KB-mapping does it with more controlling and redirecting of the learners, it is helping evoke prior knowledge through KB-map creation. This method can be used in any discipline to help learners to make connections between ideas, but the provided kit (all the important concepts and relations) always controls the process.

Reading comprehension refers to the ability to understand information presented in a written form. KBmapping method as its application helps the learners to understand the information presented in a written form, by converting the written information into a graphical form which is easily recognized and easier to scan for a specific word or general idea. Such a graphical form also allows for a more holistic understanding of the text, but at the same time controlling the process of building the learners' map by the provided kit.

The main goal of the reading task is to distinguish the important information in the text and to comprehend it. The KB-mapping method has almost the same features as the SC-mapping method but the main difference is providing the kit, which contains the important words in the text. The kit is just a list of words that support learners to distinguish the important information from the text. In other words, giving these words with the text to the learners will not be supported, rather the supported method is to use these words to find out the important information in the text, and this kind of activity can be implemented by using these words in building the learner's KB-map.

Finding the important information means not only to find the words, but more importantly it means to find the relations and integrate them together to get the whole structure of the text that contains the important information ( important information contains more than two important words). As it contains recognition of the relations between the words to complete the whole structure of the text, the important information cannot be found completely without thinking about the structure of the text. KB-mapping method reduces the learners' load in the selection process and lets the learners focus on the structuring process.

\subsection{Overview of Kit-Build concept map}

The KB-map is a special kind of concept map; just as the task to build a SC-map can be divided into two sub-tasks we have divided the task of making a concept map into two sub-tasks: 1) the "segmentation task" where parts of the concept map (nodes and links) are extracted from the text and 2) the "structuring task" where the extracted parts are integrated into a map ${ }^{(25)}$. In the KB-mapping method, learners are given a set of parts of a concept map, and then they re-build the concept map by combining the given parts. In this process, the segmentation task becomes a task of recognition of the given parts and the structuring task remains as it is.

The KB-map should be prepared beforehand by a teacher; we call this map a "goal map". The necessary and sufficient parts (kit) are generated by decomposing the goal map. The parts are provided to learners, and then the learners are required to build learner maps by connecting the parts ${ }^{(21)}$.

\subsection{KB-map system}

We have already developed a system called the "KB-map System"(20,25). It is a web application with three client applications: "Goal Map Editor", "Learner Map Builder" and "KB-map Analyzer", and one server application: "KB-map DB". The Goal Map Editor provides the teacher with an environment to edit the goal map and confirm a set of parts made from the goal map. The Learner Map Builder is an environment where learners build a map with the parts. The Goal Map Editor and Learner Map Builder have been implemented in Java. The KB-map Analyzer has functions to gather learners' maps on-line, to generate a group map (summarizing all the learners' map in one map), to adjust a goal map, and to visualize the differences between several kinds of maps. This analyzer has been implemented in Flash. The KB-map DB has a function to store and share the maps. This system has been developed in Ruby on Rails and MySQL ${ }^{(21)}$. We have improved our system to make it suitable for the reading comprehension task by adding some extra functions to help learners in building both KB-maps and SC-maps.

The new version of the Learner Map Builder has functions to add concepts and relations to the learners' 


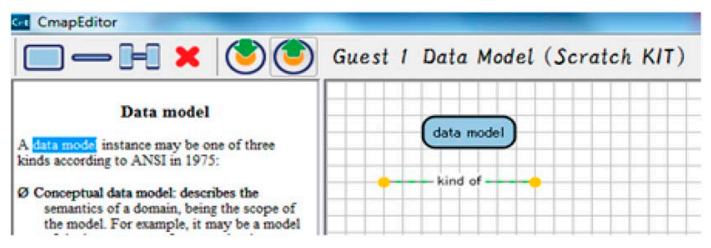

Figure 1. Part of the Learner Map Builder Interface.

map so we can use it as a computerized SC-map editor in addition to the original use of creating the KB-map from the provided kit. Moreover this version uses the Point \& Click functionality (the learners can select one or more words, and click on a creation mark to create a new node, link or relation) so learners can use it very simply to create an SC-map. Figure 1 shows a part of the Learner Map Builder interface with the new functional options. This editor has six simple functions: to create a new node, a new link, and a new relation (two nodes and a link); to delete a component; to download a map from the server; and to upload a map to the server. By using these functions the learners can use this editor to build the SC-map easily.

\section{Experiment Methodology}

For the experiment, the method of investigation is very complicated and the requirements of every session are not easily applied for a big number of participants, so we planned to do this experiment with a small number of participants in several sessions.

In the experiment, we were trying to investigate the effects of using the KB-mapping method as a supportive tool for the reading comprehension task, by comparing them with the effects of using the SC-mapping method from two viewpoints:

(1) The short-term viewpoint: we measure the understanding of the participants just after using the two methods, and compare them together.

(2) The long-term viewpoint: we measure the recalled information of the learners two weeks after using the two methods, and compare them together.

In this section, we describe the participants, outline the procedure for the whole experiment, and give the detailed procedure of one session.

\subsection{Participants}

The participants were 11 Japanese students in the
Table 1. The Average Scores for Groups A and B.

\begin{tabular}{|l|c|c|c|}
\hline $\begin{array}{l}\text { Average } \\
\text { score }\end{array}$ & TOEIC & Aptitude & $\begin{array}{c}\text { TOEIC } \\
\text { reading }\end{array}$ \\
\hline Group A & 495 & 46 & 176 \\
\hline Group B & 496.7 & 46.7 & 185.8 \\
\hline
\end{tabular}

3rd year of the information engineering course. Their TOEIC exam scores ranged from 390 to 730 , so they had different reading abilities for English texts. We prepared an aptitude test to check the learners' abilities in reading comprehension and to check if the level of text used was suitable for this experiment. This test was a simple reading task with a text, which was generated in the same way in all the sessions, and test consisted of 10 multiple choices questions. By using their TOEIC scores, TOEIC reading scores and aptitude test scores, we grouped the participants into two groups, A and B, which had almost the same average scores. Table 1 shows the average scores of the two groups.

\subsection{Procedure of the whole experiment}

The experiment was done in 6 sessions of the reading comprehension task for 6 different English texts. First, we introduced learners to the methodology of this learning process, the procedure of every session and the KB-map system. For the last 5 sessions, we started with the delayed comprehension test (DCT) of the previous session. For the DCT of the sixth session, we did it two weeks after the session. After that, we did a questionnaire. During this experiment, groups A and B each underwent Kit Build conditions (KB-conditions) 3 times and Scratch Concept map conditions (SC-conditions) 3 times. The conditions were alternated and the KBconditions group was considered to be the experimental group and the SC-conditions group was considered to be the control group. We tried to keep a balance between the different sessions regarding the texts, goal maps, and tests. We explain this later in Section 4.1.

In this experiment, the learners with SC-conditions had to find out the important information in the text; the time of segmentation and structuring was almost the same as the time needed for the recognition and structuring of KB-map building. Also they could create the node and links by a very easy and fast way because of the new function Point \& Click of the KB-editor (they only had to mark a part of the text and click on the cre- 
Table 2. The Procedure of One Session.

\begin{tabular}{|c|l|l|}
\hline Time & \multicolumn{1}{|c|}{ KB-conditions } & \multicolumn{1}{|c|}{ SC-conditions } \\
\hline 10 min & $\begin{array}{l}\text { Reading the materials (using a dictionary was al- } \\
\text { lowed) }\end{array}$ \\
\hline 20 min & $\begin{array}{l}\text { Making the KB-map by } \\
\text { using KB-editor }\end{array}$ & $\begin{array}{l}\text { Making the SC-map by } \\
\text { using KB-editor }\end{array}$ \\
\hline $5 \mathrm{~min}$ & \multicolumn{2}{|l|}{ Comprehension Test (CT) } \\
\hline $5 \mathrm{~min}$ & \multicolumn{2}{|c|}{ Delayed Comprehension Test (DCT) (2 weeks later) } \\
\hline
\end{tabular}

ation button to create a node or link). And the connecting time was the same for both methods.

But in practical use, the SC-conditions learners had to parse the text sentence by sentence to extract the concepts and to connect them with the relevant relationship at the same time.

In the experiment all the learners could create the learner's KB-maps from the kit for 3 sessions and build the learner's SC-map from the text for the other 3 sessions. Both groups had comprehended the text by using the two methods based on their CT scores. All the learners could extract appropriate concepts and build proper maps without the concepts extracted from the goal maps.

\subsection{Procedure of one session}

In the experiment, we compared the effects of using the KB-mapping method and the SC-mapping method in the reading comprehension process. We measured these effects over two stages: just after the use and after some time had passed. We designed the learning activity to be done in a limited period of time to avoid the effects of other supporting strategies. The process of one session, as shown in Table 2, consisted of four steps. In the first $10 \mathrm{~min}$, both groups were requested to read the whole text by skimming it (translating the difficult words in the text using a dictionary was allowed). Then, in the next $20 \mathrm{~min}$, the KB-conditions group was required to build the learner's KB-map of the text by using the Learner Map Builder, and in the same time the $\mathrm{SC}$-conditions group was required to build the SC-map of the text by using Learner Map Builder too. Within the building time, the learners can read the text to check their comprehension. After that both groups did the comprehension test (CT) within $5 \mathrm{~min}$ to measure their comprehension of the text. Finally, after 2 weeks both groups did the same CT again as a DCT to measure their recallable information.

\section{Experimental Materials}

In this section, we introduce two points: the preparation of the materials used and examples of the materials.

\subsection{Preparation of materials}

First we selected 6 intermediate level texts in the information engineering field to be used as materials. The participants were all students in the faculty of information engineering, so they were considered to be familiar with the topics. The texts were of the same size (word count), and taken from Wikipedia; we checked them for grammatical and semantical errors. After that, we created the corresponding KB-map (goal map) for every text, by using the Goal Map Editor. The goal map covered the main concepts and relations; and all of the goal maps had almost the same size and structure. We prepared the CTs, which were multiple choice tests with 10 questions of the same level of complexity, $80 \%$ of the comprehension questions could be answered by the goal map and the others could not. Again we checked all of the materials to be sure they did not contain any errors. Finally, we noted if the answers to questions of the CT were covered by the goal map and we marked the questions that were not covered; these were used for a detailed analysis.

\subsection{Example of materials}

In this section, we introduce one example of the materials used in the third session of our experiment.

First, the learners logged into the Learner Map Builder by using their private accounts. After that, they were requested to download the materials of the session. The KB-conditions group downloaded the text and the kit; the SC conditions group downloaded the text only.

Figure 2 shows a part of the text that was used as the original text which learners tried to comprehend it. The participants were requested to read and comprehend this text roughly within $10 \mathrm{~min}$. They could use an online dictionary to translate complex or unknown words to help them in understanding the whole text.

After that, the KB-conditions group tried to build 
the learner's KB-map, within 20 min, by using the kit that was provided by the system which generated it from the corresponding goal map. The goal map was prepared by the teacher beforehand by using the Goal Map Editor. Figure 3 shows the corresponding goal map of the text shown in Figure 2. It contains most of the information of the original text; this goal map was divided by the system to generate the kit shown in Figure 4. The SC-conditions group, on the other hand, tried to build the learner's SC-map within 20 min, using Learner Map Builder too. After the learners finished the building of the learner's maps, they were requested to upload them to the server application (KB-map DB) by using the uploading function of Learner Map Builder. KB-map DB stored all the learners' maps (KB-maps and SC-map) for later analysis.

After completing their maps, all the participants did the same CT within 5. All of these multiple choice questions asked about information included in the original text, some of them were included in the goal map and some were not. On completing the CT, they finished the day's session. They did the CT again as a DCT two weeks later.

Figure 5 shows one example of an actual learner's KB-map and Figure 6 shows one example of an actual learner's SC-map for the same written text. Figure 8 shows part of the CT test for the third session.

\section{Results}

We performed our experiment with 11 students in 6 sessions. In 3 sessions we had 5 participants as the $\mathrm{KB}$ conditions group (Group A) and 6 as the SC-conditions group (Group B), then the participants were shifted to the alternate conditions groups and we had 6 participants as the KB-conditions group and 5 as the SCconditions group. The two groups of participants alternated the conditions after every session. So, we had 6 sessions with different 6 texts, goal maps and tests, but for all the sessions participants were balanced. Table 3 shows participant details for every session, the text used, the corresponding goal map and the test which used as CT and DCT.

For every session, we compared the CT average scores, the DCT average scores and the differences (DCT-CT) for the two conditions group. We found that the KB-group had a better CT average score in 4 sessions and nearly the same average in the remaining 4 .

A language is typed if the specification of every operation
defines types of data to which the operation is applicable,
with the implication that it is not applicable to other types.
In most programming languages, dividing a number by a
string has no meaning. Most modern programming lan-
guages will therefore reject any program attempting to
perform such an operation. In some languages, the mean-
ingless operation will be detected when the program is
compiled ("static" type checking), and rejected by the
compiler, while in others, it will be detected when the
program is run ("dynamic" type checking), resulting in a
runtime exception.

Figure 2. Sample of the Text Used in the Third Session.

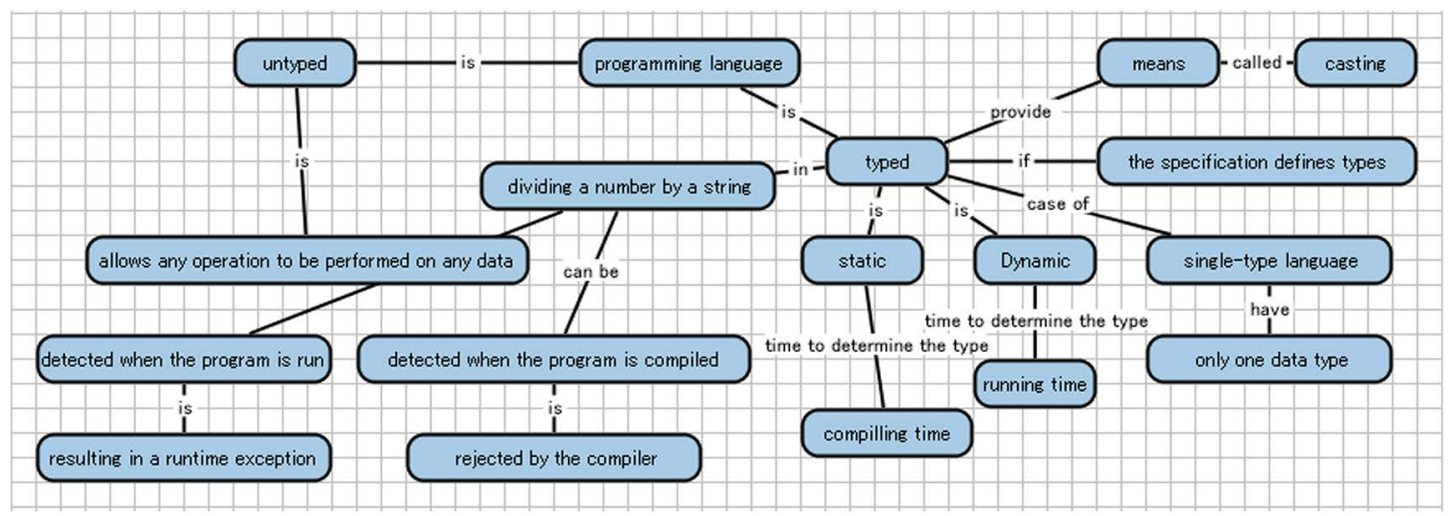

Figure 3. Goal Map of Third Session. 


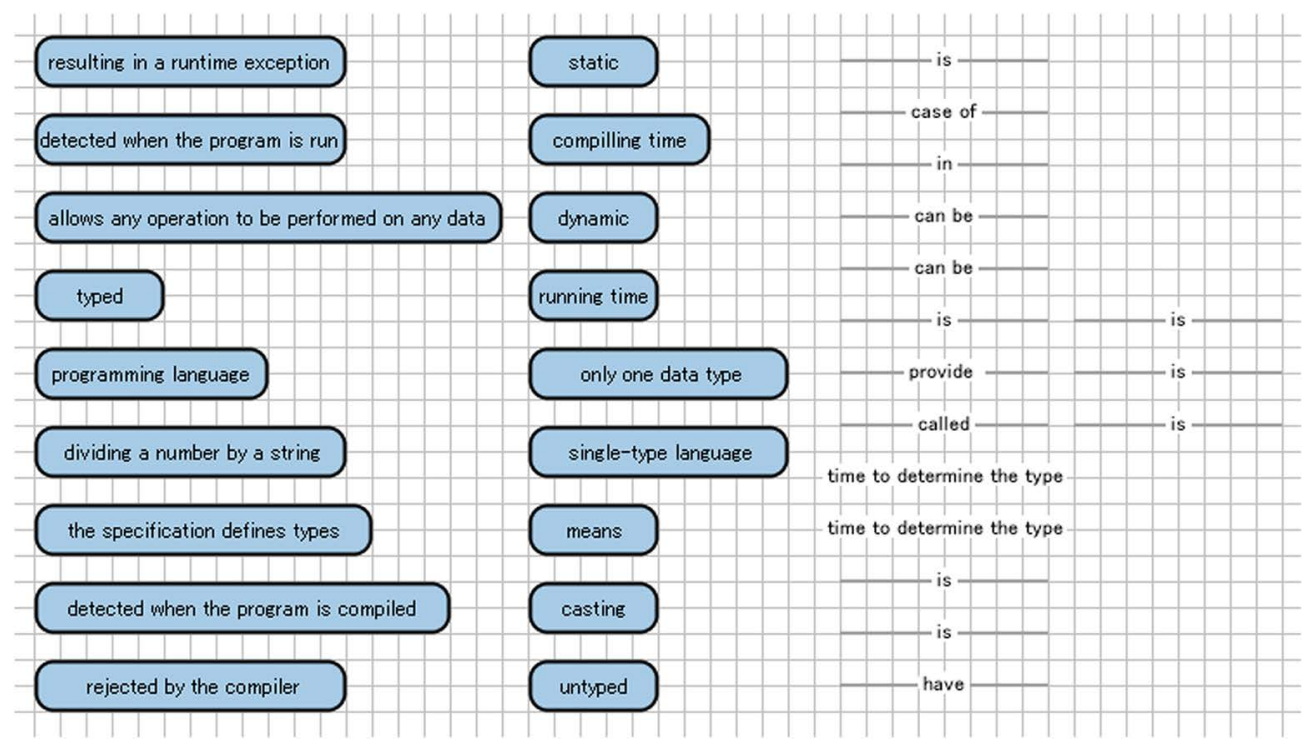

Figure 4. Kit of Third Session.

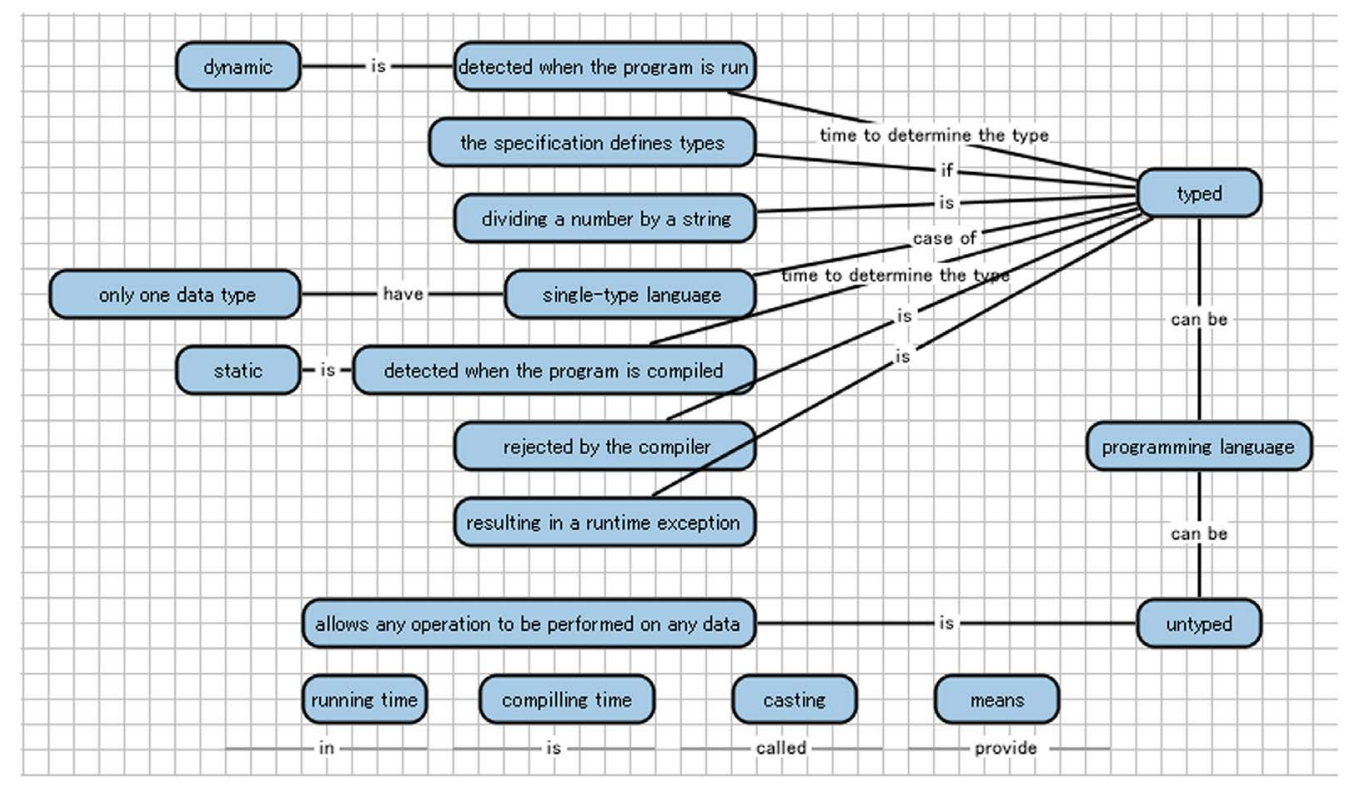

Figure 5. Example of the Learner's KB-map of the Third Session. 


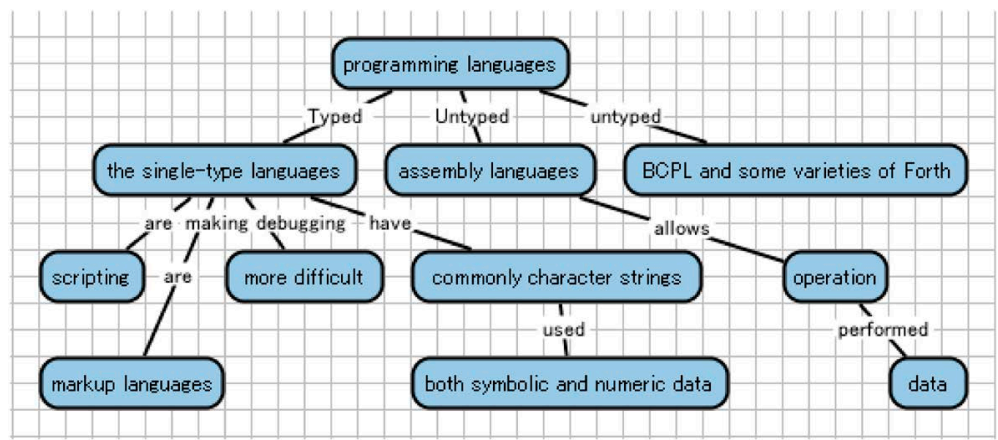

Figure 6. Example of the Learner's SC-map of the Third Session.

Q2: With typed languages, if the error of types of data is not discovered in compiling, can we discover it later?
a. can
b. cannot
c. can with conditions
d. I don't know

Q3: In untyped languages can we do an operation with two types of data?
a. can
b. cannot
c. can with conditions
d. I don't know

Q4: With untyped languages, if the error of types of data is not discovered in compiling, can we discover it later?
a. can
b. cannot
c. can with conditions
d. I don't know

Figure 7. Example Questions from the Third Session CT.

Table 3. Details of Every Session.

\begin{tabular}{|l|c|c|c|c|c|c|}
\hline Session No. & 1 & 2 & 3 & 4 & 5 & 6 \\
\hline KB-conditions & A & B & A & B & A & B \\
\hline SC-conditions & B & A & B & A & B & A \\
\hline Text & T1 & T2 & T3 & T4 & T5 & T6 \\
\hline Goal map & G1 & G2 & G3 & G4 & G5 & G6 \\
\hline Test & S1 & S2 & S3 & S4 & S5 & S6 \\
\hline
\end{tabular}

For the DCT and the difference DCT-CT (Diff) we found that the KB-group has a higher average in all the sessions. Table 4 shows the average scores of CT, DCT and (Diff) in every session for the two conditions group and the average scores for all sessions together.

The data of this experiment were a set of 6 sessions' results with 6 different texts, goal maps and tests. Usually this kind of results cannot be used in one parametric statistical analysis directly. Generally, the gathering of many tests results in one statistical analysis is a disapproval method, but for this situation, we planned all the sessions to be considered as one session as is section 4.1 Also, we confirmed that all the means for the
Table 4. Win-lose-tie Table and Binominal Test of the KBconditions Group Scores.

\begin{tabular}{|l|c|c|c|c|c|c|c|c|}
\hline $\begin{array}{l}\text { Session } \\
\text { No. }\end{array}$ & 1 & 2 & 3 & 4 & 5 & 6 & $\sum$ & $p$ (value) \\
\hline CT & 1 & 1 & 1 & 1 & 0.5 & 0.5 & 5 & 0.094 \\
\hline DCT & 1 & 1 & 1 & 1 & 1 & 1 & 6 & 0.016 \\
\hline Diff & 1 & 1 & 1 & 1 & 1 & 1 & 6 & 0.016 \\
\hline
\end{tabular}

session results were not different.

First, we tried to use the non-parametric analysis (win-lose-tie) to check which method is better. In doing so, by using Table 4, we calculated the win-lose-tie table for the KB-group scores of all the sessions for the CT, DCT and Diff.

To evaluate the results of the win-lose-tie table, we used the binominal test to calculate the probability mass function of the number of KB-mapping method winnings in all the sessions. By using the binominal test, we were looking for significance at the $5 \%$ level. We found that the KB-group won in the CT for 5 of 6 sessions (4 wins +2 ties); the probability that there were 5 successes was 
$(p(5 / 6)=0.094>0.05)$. This result indicated that the KBmapping method did not show better effects in the CT just after being used. For the DCT, we found that the KBgroup won in 6 of 6 sessions ( 6 wins); the probability that there were 6 successes was $(p(6 / 6)=0.016<0.05)$; This probability was significantly different; this result indicated that the KB-mapping method showed better effects in the DCT 2 weeks after being used. Also for the differences (DCT-CT), we found that the KB-group won in 6 of 6 sessions ( 6 wins), and the probability that there were 6 successes was $(p(6 / 6)=0.016<0.05)$; this probability was significantly different. This result indicated that the KBmapping method had better effects on recalling the comprehended information 2 weeks after being used.

\subsection{Retaining comprehension}

By comparing the average scores of the CT and the DCT for the two conditions groups in every session, we found that the experimental conditions group (KBgroup) retained more information in comparison with the control conditions group (SC-group). Figure 8 shows that for every session the average difference between DCT score and CT score of the KB-group was smaller than the average difference of the SC-group. Also from Table 3 we could confirm this result as the KB-group won in 6 sessions.

For every session we had different texts, goal maps and tests, but for all the sessions, we had the same conditions. So, we proposed to use the differences (DCT -CT) to evaluate the recalled information for the both methods. We gathered these differences for all the 6 sessions together to have a sufficient number of results for a valid statistical analysis. To confirm this approach was valid, we applied some non-parametric statistical analytical methods to check if we could use all of them together in one analysis. For the KB-group differences between the DCT and the CT scores (DCT-CT), we found by Bonferrion's method that there was no difference between any pairs of means of all session differences; and for $\mathrm{ALPHA}=0.05$, the ANOVA Test gave $p$ (value $)=$ 0.556. Also with ALPHA $=0.05$, the Friedman test gave $p($ value $)=12.945 \%$ and the Kruskal-Wallis test gave $p$ (value $)=8.62 \%$. We found that all the differences had similar distributions for $p$ (chi-square distribution). Also for the CS-group differences (DCT-CT), by using the same statistical analysis methods, we found, that there was no difference between any pairs of means of all ses-
Table 5. Difference for DCT-CT for Two Groups

\begin{tabular}{|l|c|c|}
\hline DCT-CT & KB-conditions group & SC-conditions group \\
\hline Mean & -6.129 & -14.194 \\
\hline SD & 10.679 & 15.403 \\
\hline$p$ (value) & \multicolumn{2}{|c|}{0.022} \\
\hline
\end{tabular}

sion differences and all the differences had similar distributions for $p$ (chi-square distribution).

We analyzed all the differences together by using the statistical two factor ANOVA with replications. In a simple comparison of the score means, for the KBgroup, we found that the average difference between the DCT and the CT was -6.129 , while for the SC-group, the average difference between the DCT and the CT was -14.194 . The differences of the KB-group were better than the differences of the SC-group for all the sessions. The value of the ANOVA Test $(p$ (value $)=0.022<0.05)$ indicated that, there were differences in the recalled information of the two groups. So we could say that the KB-mapping method helped the learners to retain their understanding for a long time. Table 5 shows the details of the statistical analysis.

\subsection{CT scores}

By comparing the CT average scores of the two conditions groups in all the sessions, we found that, for every session, the average score of the KB-conditions group was slightly better than the SC-conditions group; also for the whole experiment we found the same result. Figure 9 shows the average score for every session. From Table 4 we could confirm this result as the KBgroup had won in 5 of 6 sessions of the CT, but there was no significant difference: so we could say that the two methods had the same efficiency in comprehending the text just after use.

We used the statistical two factor ANOVA with replications. By using this, we found that there was no real difference between the CT scores of the two conditions groups; Table 6 showed $(p($ value $)=0.237>0.05)$ that the average score of the KB-group was slightly better than the average score of the SC-group.

\subsection{DCT scores}

By comparing the DCT scores of the two condi- 
tions groups we found that for every session the average score of the KB-conditions group was better than the $\mathrm{SC}$-conditions group; also, for the whole experiment we found the same result. Figure 10 shows the average score for every session. Again, from Table 4, we could confirm this result, as the KB-group had won in 6 of 6 sessions for the DCT, and the result was significantly different. So we could conclude that the KB-mapping method had better effects in comprehending the text deeply and in recalling the information 2 weeks later.

We used the statistical two factor ANOVA with replications. By using this, we found that there was a significant difference between the DCT scores of the two conditions groups; as shown in Table 7 $(p$ (value $)=0.005<0.01)$ the DCT average score of the KB-group was better than the DCT average score of the SC-group.

\subsection{The questionnaire results}

After we had finished the last session, the participants answered a questionnaire to evaluate KB-mapping and SC-mapping the learning methods by comparison.

Table 6. CT Scores for Two Conditions Groups.

\begin{tabular}{|l|c|c|}
\hline CT scores & KB-conditions group & SC-conditions group \\
\hline Mean & 85.313 & 80.323 \\
\hline SD & 11.986 & 16.941 \\
\hline$p$ (value) & \multicolumn{2}{|c|}{0.237} \\
\hline
\end{tabular}

Table 8 shows the results of this questionnaire. Questions (1-6, 9 \& 10) were multiple-choice questions with 5 choices which measured the participants' agreement with the mentioned point of the question. The choices were (A. Strongly agree, B. Agree, C. Neutral, D. Disagree and E. Strongly Disagree). Questions (7 \& 8) were multiple-choice questions with 3 choices which compared the two learning methods. The choices were (A. KB-mapping, B. Both are the same, C. SCmapping). To normalize the results of this questionnaire, we tried to summarize all the results and convert them to an arithmetical form: for Questions (1-6, 9 \& 10) (1 Strongly Agree, 0.5 Agree, 0 Neutral, -0.5 Disagree, and -1 Strongly Disagree); and for Questions (7 \& 8) (1 KB-mapping, 0 Both are the same and -1 SCmapping). As a summarization of the questionnaire evaluation, 0 meant neutral, positive meant agreement and negative meant disagreement, and the absolute value showed the strength of the agreement or the disagreement.

In questions $(1,4 \& 7)(2 \& 5)(3 \& 6)$, participants agreed that the KB-mapping method was useful to understand English text as was the SC-mapping. They also

Table 7. DCT Scores for Two Groups.

\begin{tabular}{|l|c|c|}
\hline DCT scores & KB-conditions group & SC-conditions group \\
\hline Mean & 79.063 & 66.1293 \\
\hline SD & 12.083 & 20.269 \\
\hline$p(t$-test $)$ & \multicolumn{2}{|c|}{0.005} \\
\hline
\end{tabular}

Table 8. Evaluation of the Proposed Methods for EFL Comprehension.

\begin{tabular}{|c|l|c|}
\hline No. & Explanation & Average agreement \\
\hline 1 & Do you think that SC-mapping was useful to understand English text? & 0.4 \\
\hline 2 & Do you think that SC-mapping was useful to answer the test after reading? & -0.5 \\
\hline 3 & Do you think that SC-mapping was useful to answer the test two weeks later? & 0.6 \\
\hline 4 & Do you think that KB-mapping was useful to understand English text? & 0.55 \\
\hline 5 & Do you think that KB-mapping was useful to answer the test after reading? & 0.1 \\
\hline 6 & Do you think that KB-mapping was useful to answer the test two weeks later? & 0.6 \\
\hline 7 & Which method was more useful to understand English text? & -0.6 \\
\hline 8 & Which method was more difficult to carry out? & 0.2 \\
\hline 9 & Do you like to use KB-mapping to understand English text? & 0.05 \\
\hline 10 & Do you like to use SC-mapping to understand English text? & \\
\hline
\end{tabular}




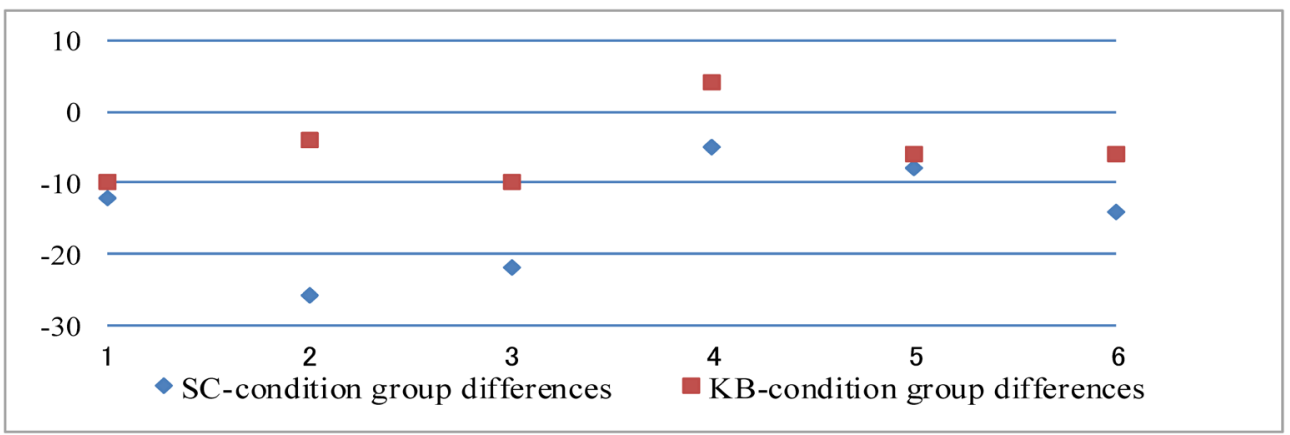

Figure 8. Average Differences of KB-conditions Group and SC-conditions Group for Each Session.

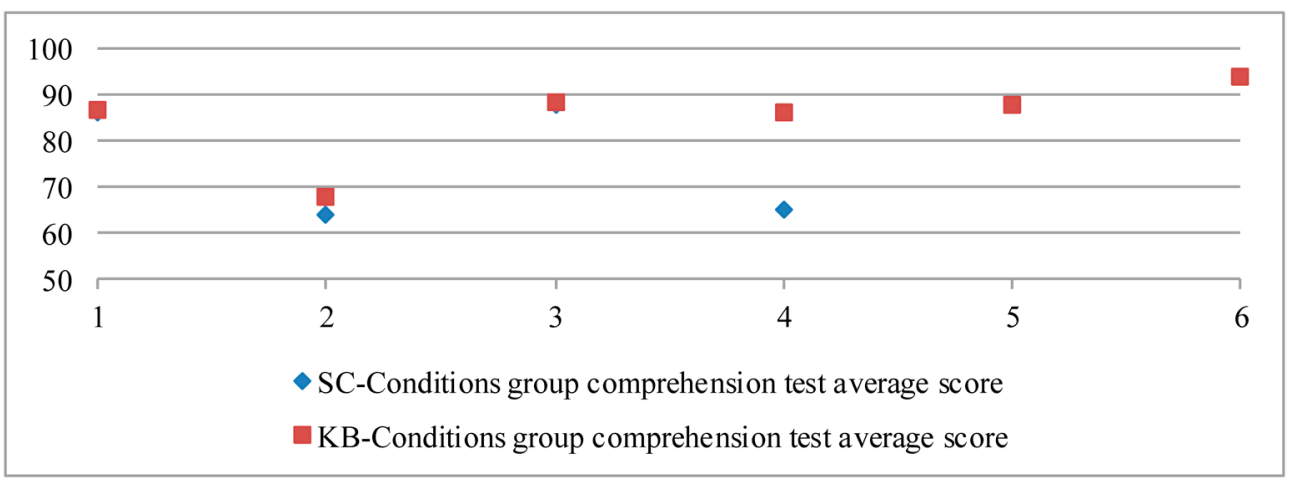

Figure 9. CT Average Score of KB-conditions Group and SC-conditions Group for Each Session.

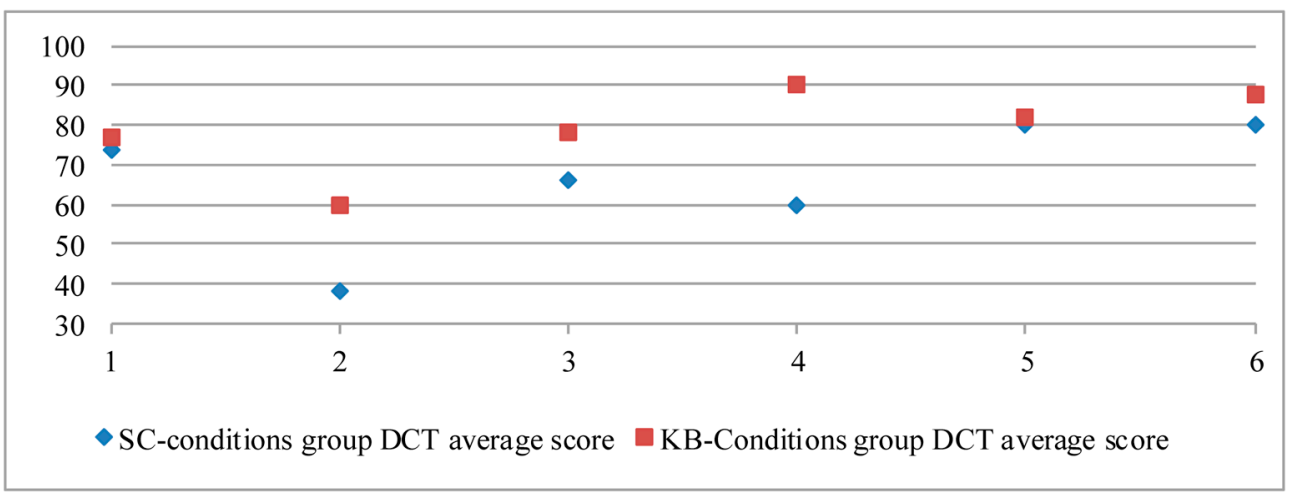

Figure 10. DCT Average Scores for KB-conditions Group and SC-conditions Group for Each Session.

said that it was useful to answer the $\mathrm{CT}$ just after the learning activity. But they agreed that the KB-mapping method was more useful to answer the DCT two weeks later. Also in questions $(8,9)$, they agreed that the KBmapping method was easier to carry out, and they liked to use the KB-mapping method in reading comprehen- 
sion task but they needed more time to do it.

In general, we could say that the learners believed that using the KB-mapping was similar to using the SCmapping for the CT, but the former was more useful for recalling the information after a while and easier to carry out. They liked to use the KB-mapping in the reading comprehension task.

\subsection{Considerations}

This experiment showed that using the KBmapping method had almost the same efficiency as using the SC-mapping method for comprehending English text just after the method use (the CT), so we could say that the two conditions groups could understand the text. And by providing a kit to the learners of the KB-group, they were supported to understand the text the same as for the SC-group.

But the KB-mapping method had better efficiency for recalling after some time had passed (the DCT). We could explain these results as because the methodology of building the learner's KB-map requires a learner to concentrate in reading the text, to distinguish two concepts that can be related, and to find the corresponding relation which can connect them together. That is because the kit (all the important concepts and relations) provided by the system forces the learner to first find all the related concepts and relations that connect them directly (in the same sentence), after that it challenges the learner to find all the non-direct relations (already provided by the system) to connect the concepts (in more than one sentence). Also this process requires the learners to understand the information in the text deeply and requires them to comprehend the text as a whole to be able to complete the whole KB-map by using all the kit. So this method helps the learner to comprehend the text deeply.

To confirm the effects of using the KB-map, by checking the effects of the other parts of the text that were not included in the goal map, we did a more detailed analysis about the "not included questions in goal map" of our experiment. For the CT, we found that the KBgroup could answer $87 \%$ of the not included questions, and the SC-group could answer $80.7 \%$. By applying the students t-test analyze to compare the two group scores in the CT, we found that there were no significant differences between them. For the DCT, we found that the KBgroup could answer $59.7 \%$ of the not included questions, and the SC-group could answer 50.6\%. By applying the
Table 9. The Not Included Question Scores.

\begin{tabular}{|l|c|c|c|}
\hline & KB-group & SC-group & $t$-test \\
\hline CT & 87.013 & 80.737 & 0.317 \\
\hline DCT & 59.740 & 50.649 & 0.380 \\
\hline
\end{tabular}

students $t$-test analyze to compare the both group scores in the DCT, we found that there were no significant differences between them. Table 9 shows the results for questions that were not included for the CT and DCT of the two conditions groups. This analysis showed that the both methods has the same efficiency for the parts of text that are not included in the goal map.

By applying the model of human memory ${ }^{(22)}$ proposed in 1968 by Atkinson and Shiffrin ${ }^{(24)}$, we could say that the required memory, for the process of constructing the learner KB-map, was the long-term memory of the Atkinson-Shiffrin Model. Moreover this process requires very high access to the memory.

On the other hand, for the SC-mapping, the learners can parse the text sentence by sentence to distinguish the important concepts in the sentences and the corresponding relation that can connect them at the same time, but there are no directions that inform the learners to continue building of the SC-map, or to challenge them to go deeply into the comprehension of the text. In the same manner, we can say that the required memory for the process of constructing the learner SCmap is the long-term memory too, the same as in the Atkinson-Shiffrin Model. But the required access to the memory is less than the KB-map situation.

So, we can explain our results by the required access to the learners; long-term memory in order to complete the student map. For the SC-group, the required access is normal, so it helped them to comprehend the text deeply, but not to memorize it long-term. But for the KB-group, the required access is high, so it helped them to comprehend most of the information that they read deeply ${ }^{(23)}$. Moreover it helped them to memorize the comprehended information long-term.

In short, using the KB-mapping method requires the learners' process meaning more deeply than they normally do with SC-mapping method.

\section{Conclusions and Future Work}

In this paper, we described the effects of using the 
KB-mapping method as a supportive tool for reading comprehension of English texts as EFL reading. Overall, from the conducted experiment, we could conclude that using KB-mapping as a learning support tool for reading comprehension was as good as using SCmapping from the short-term viewpoint, but the former was better from the long-term viewpoint.

Even given its limitations, this study provides many ideas for ways to modify teaching practices. It seems that KB-mapping can help to stimulate and challenge students to look deeper into their reading. The scope of this study was only limited to the reading comprehension. We suggest that in future research the scope should be expanded in terms of the other three important skills: writing, speaking and listening. By widening the scope, researchers can get a clear view of the utilization of KB-mapping in all aspects.

Our next goal is to investigate the effects on improving a subject's comprehension skills and acquisition of the English language, by generalizing the results with larger groups. This research will be due over a longer time period and the study will involve more participants at different levels.

\section{References}

(1) Graves, M. F., Juel, C. and Graves, B. B.: Teaching Reading in the 21st Century, Order Processing, Allyn and Bacon, IA (1998).

(2) Brown, A. L., Campione, J. C. and Day, J. D.: "Learning to Learn: On Training Students to Learn from Texts", Educational Researcher, Vol. 10, No. 2, pp. 14-21 (1981).

(3) Manoli, P. and Papadopoulou, M.: "Graphic Organizers as a Reading Strategy: Research Findings and Issues", Creative Education, Vol.3, No.3, pp. 348-356 (2012).

(4) Phakiti, A.: "Theoretical and Pedagogical Issues in ESL/ EFL Teaching of Strategic Reading", University of Sydney Papers in TESOL, Vol. 1, pp. 19-50 (2006).

(5) Salehi, A. S., Jahandar, S. and Khodabandehlou, M.: "The Impact of Concept Mapping on EFL Student's Reading Comprehension", Indian J. of Fundamental and Applied Life Sciences, Vol. 3, pp. 241-250 (2013).

(6) Piolat, A., Olive, T. and Keellogg, R. T.: "Cognitive Effort during Note Taking", Proc. Applied Cognitive Psychology, Vol. 19, No. 3, pp. 291-312 (2005)

(7) Rayner, K., Foorman, B.R., Perfetti, C.A. et al.: "How Psychological Science Informs the Teaching of Reading", Psychological Science in the Public Interest, Vol. 2, No.
2, pp. 31-74 (2001).

(8) Anderson, N.: Exploring Second Language Reading Issues and Strategies, Heinle \& Heinle Publishers, Boston, MA (1999).

(9) Sugihara, K., Osada, T., Nakata, S. et al.: "Experimental Evaluation of Kit-Build Concept Map for Science Classes in an Elementary School", Proc. of ICCE2012, Main Conference E-Book, pp. 17-24 (2012).

(10) Kalhor, M. and Shakibaei, G.: "Teaching Reading Comprehension through Concept Map", Life Science J., Vol. 4, pp. 725-731 (2012).

(11) Wikipedia: Reading Comprehension, http://en.wikipedia. org/wiki/Reading_comprehension (accessed 2013.12.10)

(12) Huber, J.A.: “A Closer Look at SQ3R", Proc. Reading Improvement, Vol. 41, No. 2, pp. 108-112 (2004).

(13) Manoli, P. and Papadopoulou, M.: "Graphic Organizers as a Reading Strategy: Research Findings and Issues", Creative Education, Vol. 3, No. 3, pp. 348-356 (2012).

(14) Yoshida, K., Osada, T., Sugihara, K. et al.: "Instantaneous Assessment of Learners' Comprehension for Lecture by Using Kit-Build Concept Map System", in Human Interface and the Management of Information. Information and Interaction for Learning, Culture, Collaboration and Business, Springer Berlin Heidelberg, pp. 175-181 (2013).

(15) Phantharakphong, P. and Pothith, S.: "Development of English Reading Comprehension by Using Concept Maps", Social and Behavioral Sciences, Vol. 116, pp. 497-501 (2014).

(16) Nesbit, J.C. and Adesope, O. O.: "Learning with Concept and Knowledge Maps: A Meta-Analysis", Review of Educational Research, Vol. 76, No.3, pp. 413-448 (2006).

(17) Alkhateeb, M., Hayashi, Y. and Hirashima, T.: "Experimental Use of KB-map to Support the Reading Comprehension of EFL", Proc. SIG-ALST-B301, Vol. 68, pp. 1-5 (2013).

(18) Alkhateeb, M., Hayashi,Y. and Hirashima, T.: "The Effects of Using Kit-Build Method to Support Reading Comprehension of EFL", in Human Interface and the Management of Information. Information and Knowledge in Applications and Services, pp. 3-11. Springer International Publishing (2014).

(19) Funaoi, H., Ishida, K. and Hirashima, T.: "Comparison of Kit-Build and Scratch-Build Concept Mapping Methods on Memory Retention", Proc. of ICCE2011, pp. 539-546 (2012)

(20) Yoshida, K., Sugihara, K., Nino, Y. et al.: "Practical Use of Kit-Build Concept Map System for Formative 
Assessment of Learners' Comprehension in a Lecture", Proc. of ICCE2013 (2013).

(21) Hirashima, T., Yamasaki, K., Fukuda, H. et al.: "Framework of Kit-Build Concept Map for Automatic Diagnosis and its Preliminary Use", Research and Practice in Technology Enhanced Learning (in press).

(22) Wikipedia: Atkinson-Shiffrin Memory Model, http:// en.wikipedia.org/wiki/Atkinson $\%$ E2\%80\%93Shiffrin memory_model (accessed 2013.12.24)

(23) Ohno, T., Hasegawa, T., Tsuruoka, T. et al.: "Short-term Plasticity and Long-term Potentiation Mimicked in Single Inorganic Synapses", Nature Materials, Vol. 10, pp. 591595 (2011).

(24) Atkinson, R.C. and Shiffrin, R.M.: "Chapter: Human Memory: A Proposed System and Its Control Processes",

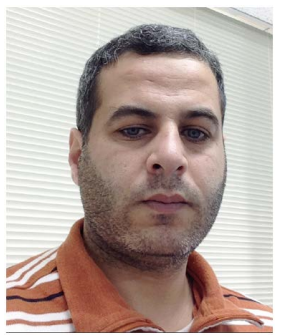

Mohammad Alkhateeb received his B.E. from Aleppo University, Syria in 2005. He received M.E. from Hiroshima University in 2012. $\mathrm{He}$ is currently a Ph.D. candidate in the Graduate School of Engineering, Hiroshima University. His research interests include AIED and TELL. $\mathrm{He}$ is a member of APSCE.

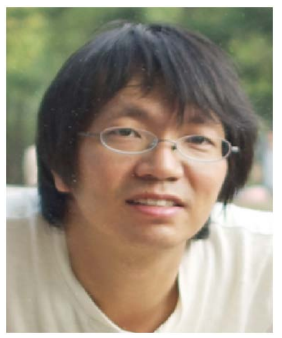

Yusuke Hayashi is an associate professor of Department of Information Engineering, Graduate School of Engineering, Hiroshima University since 2012. He received Ph.D. from Graduate School of Engineering Science, Osaka University, Japan, in 2003. He was research associate of the school of Knowledge Science, Japan Advanced Institute of Science and Technology (JAIST) from 2003 to 2005, an assistant professor of the Department of Knowledge Systems, the Institute of Scientific and Industrial Research (ISIR), Osaka University from 2005 to 2010 and an associate professor of the Information Technology Center, Nagoya University from 2010 to 2012. He has been engaged in research on Knowledge modeling, Ontological engineering, Learning engineering. He received Best paper Award of ICCE conference in 2006.
The Psychology of Learning and Motivation, Vol. 2, pp. 89-195 (1968)

(25) Hirashima, T., Yokoyama, T., Okamoto, M. et al.: "Learning by Problem-Posing as Sentence-Integration and Experimental Use", Proc. of AIED2007, pp. 254-261 (2007).

(26) Armbruster, B. B.: "Note Taking from Lectures", in Handbook of College Reading and Study Strategy Research Vol.2, eds. Flippo, R. F. and Caverly, D. C., pp. 220-248 (2009)

(27) Yamasaki, K., Fukuda, H., Hirashima, T. et al.: "Kit-Build concept map and its preliminary evaluation", The 18th International Conference on Computers in Education, pp. 290-294 (2010).

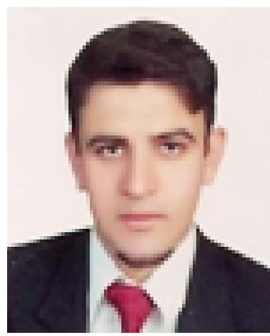

Taha Rajab received his M.A. in Sociolinguistics from the University of Leeds, UK. He received his Ph.D. from the University of York. He is currently a research fellow in the Institute for Effective Education of the University of York, UK. His research interests include applied linguistics, EFL, classroom interaction and discourse analysis. He is a member of the UK Higher Education Academy.

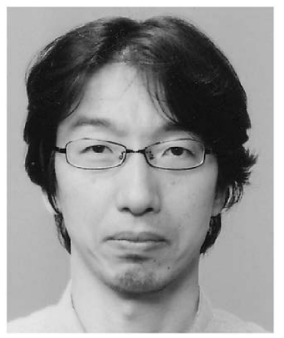

Tsukasa Hirashima received his B.E., M.E. and Ph.D. from Osaka University in 1986, 1988, and 1991 respectively. He worked at The Institute of Scientific and Industrial Research, Osaka University as a research associate and lecturer from 1991 to 1997. During 1997-2003, he worked in Graduate School of Information Engineering at Kyushu Institute of Technology as an associate professor. He has been a professor of Graduate School, Department of Information Engineering, Hiroshima University since 2004. Learning Engineering is his major research filed. He has received international awards as the Outstanding Paper Award of EDMEDIA95, the Best Paper Award of ICCE2001 \& 2002, Honorable Mention Award of AIED2009, and APSCE Distinguished Researcher Award in 2009. 